\title{
Fuzzy Dot Groups
}
${ }^{1}$ A.Solairaju
${ }^{2}$ R.Nagarajan
${ }^{3}$ P.Muruganantham
${ }^{4}$ M.Geethalakshmi

1. PG \& Research Department of Mathematics, Jamal Mohamed College, Trichy-20

2. Department of Mathematics, JJ College Of Engg \& Tech, Trichy- 09

3. Department of Mathematics, Kurinji College of Arts \& Science, Trichy-02

4. Department of Mathematics, Jayaram College of Engg \& Tech, Thuraiyur.

\begin{abstract}
:
H.Sherwood (4) introduced the concept of product of fuzzy groups (Fuzzy sets \& fuzzy systems) and its properties. We define a new class of fuzzy groups called fuzzy dot group which is weaker than the standard fuzzy group defined by Rosenfeld (2) and characterize some properties of fuzzy dot groups.
\end{abstract}

Keyword: fuzzy group, fuzzy dot group standard fuzzy group, d-fuzzy groupoid, homomorphism's, direct product.

2000AMS subject classification : 20N25, 03E72, 08 A72.

\section{INTRODUCTION}

The concept of fuzzy sets was first introduced by Zadeh (9). Rosenfeld (2) used this concept to formulate the notion of fuzzy groups. Since then, many other fuzzy algebraic concepts based on the Rosenfeld's fuzzy groups were developed. Anthony and Sherwood (1) redefined fuzzy groups in terms of t- norm which is replaced the min operations of Rosenfeld's definition. Some properties of these redefined fuzzy groups, which we call t- fuzzy groups, have been developed by Sherwood (4), sessa (3), sidky and misherf (5). However the definition of t- fuzzy groups seems to be too general. A.Solairaju and R.Nagarajan (6) investigate the characterizations of weaker groups in terms of Rosenfeld groups. We define a new class of fuzzy group called fuzzy dot group which is weaker than the fuzzy groups defined by Rosenfeld's (2), and characterize some properties of fuzzy dot groups.

\section{PRELIMINARIES}

Definition 2.1: A function ' $A$ ' from a set $X$ to the closed unit interval $[0,1]$ in $U$ is called a fuzzy set in $X$, for every $x \in A$, $\mathrm{A}(\mathrm{x})$ is called membership grade of $\mathrm{x}$ in A. The set $\quad\{\mathrm{x} \varepsilon$ $\mathrm{A} / \mathrm{A}(\mathrm{x})>0\}$ is called the support of $\mathrm{A}$ and it is denoted by $\operatorname{supp}(\mathrm{A})$. For fuzzy sets $\lambda$ and $\mu$ in a set $X$, then $\lambda \circ \mu$ has been defined in most articles by

$$
\begin{aligned}
(\lambda \circ \mu)(x) & =\sup \min \{\lambda(a), \mu(b)\}, & & \text { if } a b=x \\
& a b=x & & \\
& =0 & & \text { if } a b \neq x
\end{aligned}
$$

We weaker this definition as follows.

Definition 2.2: Let $X$ be a set and let $\lambda, \mu$ be two fuzzy sets in $\mathrm{X}, \lambda \circ \mu$ is defined by

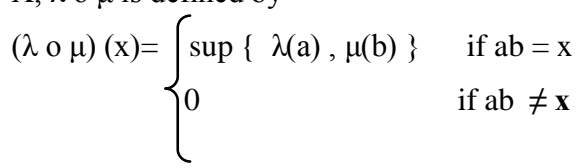

Definition 2.3: Let $X$ be a group, we define $\lambda^{-1}$ by $\lambda^{-1}(x)=$ $\lambda\left(\mathrm{x}^{-1}\right)$ for $\mathrm{x} \varepsilon \mathrm{X}$. The standard definition of a fuzzy group by Rosenfeld(2) is that a fuzzy set ' $A$ ' in a group $X$ is a fuzzy group iff $\mathrm{A}(\mathrm{xy}) \geq \min \{\mathrm{A}(\mathrm{x}), \mathrm{A}(\mathrm{y})\}$ and $\mathrm{A}\left(\mathrm{x}^{-1}\right)=\mathrm{A}(\mathrm{x})$ for all $\mathrm{x}, \mathrm{y} \varepsilon \mathrm{X}$ we weaken this definition as follows.

Definition 2.4: Let ' $S$ ' be a groupoid. A function $A: S \rightarrow$ $[0,1]$ is a dot groupoid in $S$ iff for every $x, y$ in $S$, (FDG1) $\mathrm{A}(\mathrm{xy}) \geq \mathrm{A}(\mathrm{x}) \cdot \mathrm{A}(\mathrm{y})$, we denote a dot fuzzy groupoid by a d- fuzzy groupoid. If $X$ is a group, a fuzzy dot groupoid ' $A$. in $\mathrm{X}$ iff for $\mathrm{x} \varepsilon \mathrm{X}$, (FDG2) $\quad \mathrm{A}\left(\mathrm{x}^{-1}\right)=\mathrm{A}(\mathrm{x})$, we denote a dot fuzzy group $X$ by a $d$ - fuzzy group . Since $\min (a, b) \geq a b$, our definition of a d- fuzzy group is weaker than the standard definition by Rosenfeld(2). It is easy to see that if $G$ is fuzzy group in a group $\mathrm{X}$ and ' $\mathrm{e}$ ' is the identity of $\mathrm{X}, \quad \mathrm{G}(\mathrm{e}) \geq$ 
$\mathrm{G}(\mathrm{x})$ for all $\mathrm{x} \varepsilon \mathrm{X}$. If ' $\mathrm{G}$ ' is a d-fuzzy group in a group $\mathrm{X}, \mathrm{G}(\mathrm{e})$ $=G\left(x x^{-1}\right) \geq G(x) G\left(x^{-1}\right)=[G(x)]^{2}$ for all $x \in X$

\section{SOME PROPERTIES OF D- FUZZY GROUPS}

Proposition 3.1: Let 'A' be a fuzzy subset in a group $X$ such that $\mathrm{A}(\mathrm{e})=1$, where ' $\mathrm{e}$ ' is the identity of $\mathrm{X}$ then ' $\mathrm{A}$ ' is a $\mathrm{d}$ fuzzy group iff $\mathrm{A}\left(\mathrm{xy}^{-1}\right) \geq \mathrm{A}(\mathrm{x}) \cdot \mathrm{A}(\mathrm{y})$ for all $\mathrm{x}, \mathrm{y} \varepsilon \mathrm{X}$.

Proof: suppose ' $A$ ' is d- fuzzy group. Then $A\left(x y^{-1}\right) \geq A(x)$ $\cdot A\left(x^{-1}\right)=A(x) \cdot A(y)$.Suppose $A\left(x y^{-1}\right) \geq A(x) \cdot A(y)$. Then $\mathrm{A}\left(\mathrm{x}^{-1}\right)=\mathrm{A}\left(\mathrm{ex}^{-1}\right) \geq \mathrm{A}(\mathrm{e}) \cdot \mathrm{A}\left(\mathrm{x}^{-1}\right) \geq \mathrm{A}(\mathrm{x})=\mathrm{A}(\mathrm{ex}) \geq \mathrm{A}(\mathrm{e}) \cdot \mathrm{A}(\mathrm{x})$ $=\mathrm{A}\left(\mathrm{x}^{-1}\right)$ That is $\mathrm{A}(\mathrm{x})=\mathrm{A}\left(\mathrm{x}^{-1}\right)$ and $\mathrm{A}\left(\mathrm{xy}^{-1}\right)=\mathrm{A}\left(\mathrm{x}\left(\mathrm{y}^{-1}\right)\right) \geq \mathrm{A}(\mathrm{x})$ $\cdot \mathrm{A}\left(\mathrm{y}^{-1}\right)=\mathrm{A}(\mathrm{x}) \cdot \mathrm{A}(\mathrm{y})$.

Definition 3.2: Let ' $A$ ' be a d- fuzzy groupoid in a group $X$ such that $A(a)=A\left(a^{-1}\right)$. Let $e \lambda: X \rightarrow X$ be identity defined by $\mathrm{e}_{\lambda}(\mathrm{x})=\mathrm{x} \lambda$ for all $\mathrm{x} \varepsilon A$. similar we can define the left identity of $A$.

Proposition 3.3: If the $d$ - fuzzy groupoid ' $A$ ' on $X$ has left identity $e_{\lambda}$ and a right identity $e_{\mu}$ then $e_{\lambda}=e_{\mu}$.

Proof: $e_{\lambda}(A)(x)=\sup A(z)=A\left(x^{-1} a\right)$

$$
\begin{gathered}
z \in e_{\lambda}^{-1}(x) \\
\geq A(x) \cdot A\left(a^{-1}\right) \\
=A(x) \\
=A\left(x a^{-1} a\right) \geq A\left(x a^{-1}\right) \cdot A(a)=A\left(x a^{-1}\right) \\
=e_{\lambda}(A)(x)
\end{gathered}
$$

Thus $e_{\lambda}(A)(x) \geq A(x) \geq e_{\lambda}(A)(x)$. That is $e_{\lambda}(A)=A$. similarly we may show $e_{\mu}(A)=A$.

Definition 3.4: Let $\mathrm{f}: \mathrm{G} \rightarrow \mathrm{G}^{1}$ be a homomorphism of d-fuzzy groups. For any fuzzy set $A \varepsilon G^{1}$ we define a new fuzzy set $A^{f}$ in $G$ by $A^{f}(x)=\operatorname{Af}(x)$ for all $x \in G$, and $f\left(x^{-1}\right)$ $=f(x)=(f(x))^{-1}$.

Definition 3.5. Let $A$ and $B$ be two fuzzy subsets of $X$ then the direct product $\mathrm{A} \times \mathrm{B}$ is defined by $(\mathrm{A} \times \mathrm{B})(\mathrm{x}, \mathrm{y})=\min$ $\{\mathrm{A}(\mathrm{x}), \mathrm{B}(\mathrm{y})\}$ and $(\mathrm{x}, \mathrm{y}) \cdot(\mathrm{z}, \mathrm{p})=(\mathrm{xz}, \mathrm{yp})$ for all $\mathrm{x}, \mathrm{y}, \mathrm{z}, \mathrm{p}$ in $\mathrm{X}$.

Proposition 3.6: Let $G$ and $G^{1}$ be groups and $f$ a homomorphism from $G$ onto $G^{1}$, (i) if $A$ is d- fuzzy group of $G^{1}$ then $A^{f}$ is d- fuzzy group of $G$.(ii) if $A^{f}$ is d- fuzzy group of $G$ then $A$ is d-fuzzy group of $G^{1}$.
Proof : (i) Let x,y $\varepsilon$ G, we have (FDG1) $A^{f}(x y)=A f(x y)=$ A

$\geq \operatorname{Af}(\mathrm{x}) \cdot \operatorname{Af}(\mathrm{y})=\mathrm{A}^{\mathrm{f}}(\mathrm{x}) \cdot \mathrm{A}^{\mathrm{f}}(\mathrm{y})$.

(FDG2) $A^{f}\left(x^{-1}\right)=\operatorname{Af}\left(x^{-1}\right)=\operatorname{Af}(x)=A^{f}(x)$

$A^{f}$ is d- fuzzy group of $G^{1}$.

(ii) For any $\mathrm{x}, \mathrm{y} \varepsilon \mathrm{G}^{1}$, There exists $\mathrm{a}, \mathrm{b} \varepsilon \mathrm{G}$ such that $\mathrm{f}(\mathrm{a})=\mathrm{x}$ and $\mathrm{f}(\mathrm{b})=\mathrm{y}$.

(FDG1) $\mathrm{A}(\mathrm{xy})=\mathrm{A}(\mathrm{f}(\mathrm{a}) \mathrm{f}(\mathrm{b}))=\operatorname{Af}(\mathrm{ab})=\mathrm{A}^{\mathrm{f}}(\mathrm{ab}$

$$
\geq A^{f}(a) \cdot A^{f}(b)=\operatorname{Af}(a) \cdot A f(b)=A(x) \cdot A(
$$

(FDG2) $\mathrm{A}\left(\mathrm{x}^{-1}\right)=\mathrm{A}\left(\mathrm{f}\left(\mathrm{a}^{-1}\right)\right)=\operatorname{Af}\left(\mathrm{a}^{-1}\right)=\mathrm{A}^{\mathrm{f}}\left(\mathrm{a}^{-1}\right)=\mathrm{A}^{\mathrm{f}}(\mathrm{a})=\operatorname{Af}(\mathrm{a})=$ $\mathrm{A}(\mathrm{x})$

A is d- fuzzy group of $\mathrm{G}^{1}$.

Proposition 3.7: If $A$ and $B$ be $d$ - fuzzy groups of $G_{1}$ and $G_{2}$ respectively then $A \times B$ is d- fuzzy group of $G_{1} \times G_{2}$.

Proof: Let $\left(a_{1}, b_{1}\right),\left(a_{2}, b_{2}\right) \varepsilon G_{1} \times G_{2}$

(FDG1) $\mathrm{A} \times \mathrm{B}\left(\left(\mathrm{a}_{1}, \mathrm{~b}_{1}\right)\left(\mathrm{a}_{2}, \mathrm{~b}_{2}\right)\right)=(\mathrm{A} \times \mathrm{B})\left(\mathrm{a}_{1} \mathrm{a}_{2}, \mathrm{~b}_{1}=\min \{\right.$ $\mathrm{A}\left(\mathrm{a}_{1} \mathrm{a}_{2}\right), \mathrm{B}\left(\mathrm{b}_{1} \mathrm{~b}\right.$

$\geq \min \left\{\mathrm{A}\left(\mathrm{a}_{1}\right) \cdot \mathrm{A}\left(\mathrm{a}_{2}\right), \mathrm{B}\left(\mathrm{b}_{1}\right) \cdot \mathrm{B}\left(\mathrm{b}_{2}\right)\right\}$

$\geq \min \left\{\mathrm{A}\left(\mathrm{a}_{1}\right) \cdot \mathrm{B}\left(\mathrm{b}_{1}\right), \mathrm{A}\left(\mathrm{a}_{2}\right) \cdot \mathrm{B}\left(\mathrm{b}_{2}\right)\right\}$

$\geq \min \left\{\left\{\mathrm{A}\left(\mathrm{a}_{1}\right), \mathrm{B}\left(\mathrm{b}_{1}\right)\right\}, \min \left\{\mathrm{A}\left(\mathrm{a}_{2}\right), \mathrm{B}\left(\mathrm{b}_{2}\right)\right\}\right\}$

$\geq \mathrm{A} \times \mathrm{B}\left(\mathrm{a}_{1}, \mathrm{~b}_{1}\right) \quad \mathrm{A} \times \mathrm{B}\left(\mathrm{a}_{2}, \mathrm{~b}_{2}\right)$

(FDG2) $\mathrm{A} \times \mathrm{B}\left(\mathrm{a}_{1}, \mathrm{~b}_{1}\right)^{-1}=\mathrm{A} \times \mathrm{B}\left(\mathrm{a}_{1}{ }^{-1}, \mathrm{~b}_{1}{ }^{-1}\right)$

$=\min \left\{\mathrm{A}\left(\mathrm{a}_{1}^{-1}\right), \mathrm{B}\left(\mathrm{b}_{1}^{-1}\right)\right\}$

$=\min \left(\mathrm{A}\left(\mathrm{a}_{1}\right), \mathrm{B}\left(\mathrm{b}_{1}\right)\right\}$ 
Proposition 3.8: If $A$ and $B$ be $d$ - fuzzy groups of $G_{1}$ and $G_{2}$ respectively then $A \times B$ is $\quad d$ - fuzzy group of $G_{1} \times G_{2}$.

Proof: Let $\left(a_{1}, b_{1}\right),\left(a_{2}, b_{2}\right) \varepsilon G_{1} \times G_{2}$

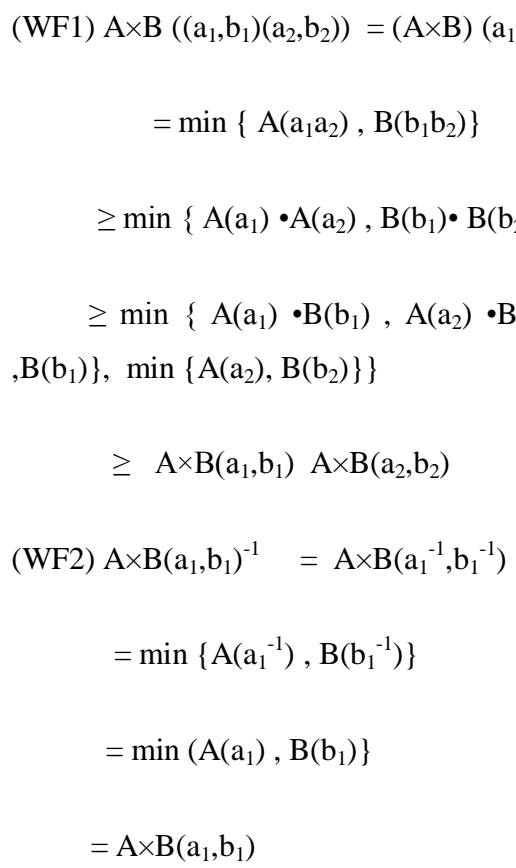

Corollary 3.9: If $A_{1}, A_{2}, \ldots A_{n}$ are $d$ - fuzzy groups of $G_{1}$, $G_{2} \ldots G_{n}$ respectively then $A_{1} \times A_{2} \times \ldots \ldots A_{n}$ is d-fuzzy groups of $\mathrm{G}_{1} \times \mathrm{G}_{2} \times \ldots \ldots \mathrm{G}_{\mathrm{n}}$.

Proof: This result can easily show by induction method.

Proposition 3.10: Let $A$ and $B$ be fuzzy subsets of $G_{1}$ and $G_{2}$ respectively such that $A \times B$ is a d- fuzzy group of $G_{1} \times G_{2}$ then $A$ and $B$ is d- fuzzy group of $G_{1}$ and $G_{2}$ respectively.

\section{Proof:}

$(\mathrm{A} \times \mathrm{B})\left(\mathrm{e}_{1}, \mathrm{e}_{2}\right)=\min \left(\mathrm{A}\left(\mathrm{e}_{1}\right), \mathrm{A}\left(\mathrm{e}_{2}\right)\right\} \geq(\mathrm{A} \times \mathrm{B})(\mathrm{x}, \mathrm{y})$ for all $(\mathrm{x}, \mathrm{y})$ $\varepsilon \mathrm{G}_{1} \times \mathrm{G}_{2}$, then

$\mathrm{A}(\mathrm{x}) \leq \mathrm{A}\left(\mathrm{e}_{1}\right)$ or $\mathrm{B}(\mathrm{y}) \leq \mathrm{B}\left(\mathrm{e}_{2}\right)$. If $\mathrm{A}(\mathrm{x}) \leq \mathrm{A}\left(\mathrm{e}_{1}\right)$ then $\mathrm{A}(\mathrm{x}) \leq$ $\mathrm{B}\left(\mathrm{e}_{2}\right)$ or $\mathrm{B}(\mathrm{y}) \leq \mathrm{B}\left(\mathrm{e}_{2}\right)$.

Let $\mathrm{A}(\mathrm{x}) \leq \mathrm{B}\left(\mathrm{e}_{2}\right)$. Then for all $\mathrm{x}, \mathrm{y} \varepsilon \mathrm{G}(\mathrm{A} \times \mathrm{B})\left(\mathrm{x}, \mathrm{e}_{2}\right)=\mathrm{A}(\mathrm{x})$

(FDG1) $\mathrm{A}(\mathrm{xy}) \quad=(\mathrm{A} \times \mathrm{B})\left(\mathrm{xy}, \mathrm{e}_{2}\right)$

$=(\mathrm{A} \times \mathrm{B})\left(\left(\mathrm{x}, \mathrm{e}_{2}\right)\left(\mathrm{y}, \mathrm{e}_{2}\right)\right)$

$\geq(\mathrm{A} \times \mathrm{B})\left(\mathrm{x}, \mathrm{e}_{1}\right) \cdot(\mathrm{A} \times \mathrm{B})\left(\mathrm{y}, \mathrm{e}_{2}\right)$ $\geq \mathrm{A}(\mathrm{x}) \cdot \mathrm{A}(\mathrm{y})$

(FDG2) $\mathrm{A}\left(\mathrm{x}^{-1}\right) \quad=(\mathrm{A} \times \mathrm{B})\left(\mathrm{x}^{-1}, \mathrm{e}_{2}\right)$

$=(\mathrm{A} \times \mathrm{B})\left(\mathrm{x}^{-1}, \mathrm{e}_{2}^{-1}\right)$

$=(\mathrm{A} \times \mathrm{B})\left(\mathrm{x}, \mathrm{e}_{2}\right)^{-1}$

$=(\mathrm{A} \times \mathrm{B})\left(\mathrm{x}, \mathrm{e}_{2}\right)$

$=\mathrm{A}(\mathrm{x})$ Therefore $\mathrm{A}$ is $\mathrm{w}-$ fuzzy

group of G.

Now suppose that $A(x) \leq B\left(e_{2}\right)$ is not true for all $x \varepsilon G_{1}$. If $A(x) \geq B\left(e_{2}\right)$ there exists $x \in G_{1}$, then $B(y) \leq B\left(e_{2}\right)$ for all y $\varepsilon$ $\mathrm{G}_{2}$. Therefore

$(A \times B)\left(e_{1}, y\right)=B(y)$ for all y $\varepsilon G_{2}$. Similarly for all $x, y \varepsilon G_{2}$,

$$
\begin{aligned}
\mathrm{B}(\mathrm{xy}) & =(\mathrm{A} \times \mathrm{B})\left(\mathrm{e}_{1}, \mathrm{xy}\right) \\
& =(\mathrm{A} \times \mathrm{B})\left(\left(\mathrm{e}_{1}, \mathrm{x}\right)\left(\mathrm{e}_{2}, \mathrm{y}\right)\right) \\
\geq & (\mathrm{A} \times \mathrm{B})\left(\mathrm{e}_{1}, \mathrm{x}\right) \cdot(\mathrm{A} \times \mathrm{B})\left(\mathrm{e}_{1}, \mathrm{y}\right) \\
& =\mathrm{B}(\mathrm{x}) \cdot \mathrm{B}(\mathrm{y})
\end{aligned}
$$

And

$$
\begin{aligned}
\mathrm{B}\left(\mathrm{x}^{-1}\right) & =(\mathrm{A} \times \mathrm{B})\left(\mathrm{e}^{1}, \mathrm{x}^{-1}\right) \\
& =(\mathrm{A} \times \mathrm{B})\left(\mathrm{e}_{1}^{-1}, \mathrm{x}^{-1}\right) \\
& =(\mathrm{A} \times \mathrm{B})\left(\mathrm{e}_{1}, \mathrm{X}\right)^{-1} \\
& =(\mathrm{A} \times \mathrm{B})\left(\mathrm{e}_{1}, \mathrm{x}\right)=\mathrm{B}(\mathrm{x}) \text { hence } \mathrm{B} \text { is } \mathrm{d}-
\end{aligned}
$$

fuzzy group of $\mathrm{G}_{2}$ consequently either $A$ or $B$ is $d$ - fuzzy group of $\mathrm{G}_{1}$ or $\mathrm{G}_{2}$ respectively.

Definition 3.11: Let $f: G \rightarrow G^{1}$ be a group homomorphisms and ' $A$ ' be $d$ - fuzzy group of $G^{1}$ then $\operatorname{Af}(x)=(A \circ f)(x)=f$ ' ${ }^{1}(\mathrm{~A})(\mathrm{x})$

Proposition3.12: Let $\mathrm{f}: \mathrm{G} \rightarrow \mathrm{G}^{1}$ be a group homomorphism and let ' $A$ ' be a d- fuzzy group of $G^{1}$ then $f^{-1}(A)$ is d- fuzzy group of $\mathrm{G}$.

Proof: Let $\mathrm{x}, \mathrm{y} \varepsilon \mathrm{G}$, we have 
(FDG1)

$$
\mathrm{f}^{-1}(\mathrm{~A})(\mathrm{xy})=(\text { Aof })(\mathrm{xy})
$$$$
=\operatorname{Af}(x y)
$$$$
=A(f(x) f(y))
$$$$
\geq \operatorname{Af}(x) \cdot \operatorname{Af}(y)
$$$$
\geq(\text { Aof })(x) \bullet(\text { Aof })(y)
$$

$$
\geq f^{1}(A)(x) \cdot f^{-1}(A)(y)
$$

$$
\begin{aligned}
& (\mathrm{FDG} 2) \quad \mathrm{f}^{-1}(\mathrm{~A})\left(\mathrm{x}^{-1}\right)=(\text { Aof })\left(\mathrm{x}^{-1}\right)=\operatorname{Af}\left(\mathrm{x}^{-1}\right)= \\
& \mathrm{A}(\mathrm{f}(\mathrm{x}))=\mathrm{f}^{-1}(\mathrm{~A})(\mathrm{x})
\end{aligned}
$$

Proposition 3.13: Let A be a d- fuzzy group of group G and $A^{*}$ be a fuzzy set in $G$ defined by $A^{*}(x)=A(x)+1-A(e)$ for all $x \in G$. Then $A *$ is d- fuzzy group of $G$ containing $A$.

Proof: for $\mathrm{x}, \mathrm{y} \varepsilon \mathrm{G}$, we have

(FDG1)

$$
\mathrm{A}^{*}(\mathrm{xy}) \quad=\mathrm{A}(\mathrm{xy})+1-\mathrm{A}(\mathrm{e})
$$

$$
\geq(\mathrm{A}(\mathrm{x}) \cdot \mathrm{A}(\mathrm{y}))+1-\mathrm{A}(\mathrm{e})
$$

$\geq(\mathrm{A}(\mathrm{x})+1-\mathrm{A}(\mathrm{e})) \cdot(\mathrm{A}(\mathrm{y})+1-\mathrm{A}(\mathrm{e}))$

$$
\geq \mathrm{A}^{*}(\mathrm{x}) \cdot \mathrm{A}^{*}(\mathrm{y})
$$

$$
\begin{aligned}
(\mathrm{FDG} 2) \mathrm{A}^{*}\left(\mathrm{x}^{-1}\right)=\mathrm{A}\left(\mathrm{x}^{-1}\right)+1-\mathrm{A}(\mathrm{e}) \\
=\mathrm{A}(\mathrm{x})+1-\mathrm{A}(\mathrm{e}) \\
=\mathrm{A}^{*}(\mathrm{x})
\end{aligned}
$$

A* is d- fuzzy group of $\mathrm{G}$ containing A.

Proposition3.14 : Let $\langle A\rangle=\left\{A, A^{1}, A^{2},----A^{P}----E\right\}$ then $\mathrm{U} \mathrm{A}^{\mathrm{P}}=\mathrm{A}$

$\infty$

$$
\mathrm{p}=1
$$$$
\text { and } \cap \mathrm{A}^{\mathrm{P}}=\mathrm{E}
$$

$$
\mathrm{p}=1
$$

Proof: Let $\mathrm{x} \varepsilon \mathrm{G} \quad$ we have $\quad \mathrm{A} \subset \quad \mathrm{U} \mathrm{A}^{\mathrm{P}}$ (i)

$$
\mathrm{p}=1
$$

(since $\left.\mathrm{A}(\mathrm{x}) \leq \mathrm{U} \mathrm{A}^{\mathrm{p}}(\mathrm{x})\right)$

$\mathrm{UA}^{\mathrm{P}}(\mathrm{x})=\max \left\{\mathrm{A}(\mathrm{x}), \mathrm{A}^{2}(\mathrm{x}),-----\right\}$
$=\mathrm{A}(\mathrm{x}) \quad\left(\right.$ since $\mathrm{A}(\mathrm{x}) \leq(\mathrm{A}(\mathrm{x}))^{\mathrm{p}}$ for all natural number $\mathrm{p}$.

$\infty$ $\infty$

$\mathrm{U} \mathrm{A}^{\mathrm{p}} \subset \mathrm{A}$ (ii) from (i) and (ii) $\quad \mathrm{U}^{\mathrm{p}}=\mathrm{A}$.

Also we have to

$\mathrm{p}=1$

$\mathrm{p}=1$

$\infty$

Show that $E=\cap A^{p}$. Let e $\varepsilon A^{P}$ implies that $(e, 1) \varepsilon A^{p}$ for all p.

$$
\mathrm{p}=1 \quad \infty
$$

Implies that $(\mathrm{e}, 1) \quad \cap \mathrm{A}^{\mathrm{p}}$

$$
\infty \mathrm{p}=1
$$

Therefore $\mathrm{E} \subset \cap \mathrm{A}^{\mathrm{p}}$ (iii)

$$
\infty \quad \mathrm{p}=1
$$

Let $\mathrm{x} \varepsilon \cap \mathrm{A}^{\mathrm{p}}$ implies that $\mathrm{x} \varepsilon \mathrm{A}^{\mathrm{p}}$ for all $\mathrm{p}$.

$$
\mathrm{p}=1
$$

$\infty$

$\cap A^{P}(x)=\min \left\{A(x), A^{2}(x)-----\right\}$

$\mathrm{p}=1$

$$
\begin{array}{ll}
=0 & \text { if } \mathrm{x} \neq \mathrm{e} \\
=1 & \text { if } \mathrm{x}=\mathrm{e}
\end{array}
$$

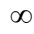

$\mathrm{x} \varepsilon \cap \mathrm{A}^{\mathrm{p}}$ implies that $\mathrm{x}=\mathrm{e}$ thus $\cap \mathrm{A}^{\mathrm{p}} \subset \mathrm{E}$

- (iv) hence

$\mathrm{p}=1 \quad \infty \quad \mathrm{p}=1$

From (iii) and (iv) $\mathrm{E}=\cap \mathrm{A}^{\mathrm{P}}$

$$
\mathrm{p}=1
$$

Proposition3.15: Let $\mathrm{A}$ be a d-fuzzy group, then $\mathrm{A} \supset \mathrm{A}^{2} \supset$ $\mathrm{A}^{3} \ldots \ldots \ldots . . \supset \mathrm{A}^{\mathrm{p}} \ldots \ldots . . \mathrm{E}$.

Proof: It is known that $\mathrm{A}(\mathrm{a}) \varepsilon[0,1]$, hence $\mathrm{A}(\mathrm{a}) \geq \mathrm{A}(\mathrm{a})^{2}$, $\mathrm{A}\left(\mathrm{a}^{2}\right) \geq\left(\mathrm{A}\left(\mathrm{a}^{2}\right)\right)^{2}$ $A\left(a^{n}\right) \geq\left(A\left(a^{n}\right)\right)^{2}$ by using the definition of fuzzy subsets, this gives that $\mathrm{A} \supset \mathrm{A}^{2}$. By generalizing it for any natural numbers $i$ and $j$ with $i \leq j$. we obtain $\left(A_{i}(a)\right)^{i} \geq\left(A_{j}(a)\right)^{j}, \quad\left(A_{i}\left(a^{2}\right)\right)^{i} \geq\left(A_{j}\left(a^{2}\right)^{j}\right.$, $\left(\mathrm{A}_{\mathrm{i}}\left(\mathrm{a}^{\mathrm{n}}\right)\right)^{\mathrm{i}}$ $\left.\geq A_{j}\left(a^{n}\right)\right)^{j}$

So $A^{i} \supset A^{j}$ for any natural numbers $i$ and $j$ with $i \leq j$ which means that $A \supset A^{2} \supset A^{3} \ldots . \supset A^{p n} \ldots$ finally we get $E=\cap A^{p}$ which is immediate from proposition (3.14). Since

$$
\begin{aligned}
& \text { Lt } \mathrm{A}(\mathrm{a})^{\mathrm{p}}=1 \quad \text { if } \mathrm{a}=\mathrm{e} . \\
& \mathrm{n} \rightarrow \infty \quad=0 \quad \text { if } \mathrm{a} \neq \mathrm{e} \quad \text { we then obtain the required } \\
& \text { relations. }
\end{aligned}
$$




\section{CONCLUSION}

H.Sherwood introduced the concept of product of fuzzy groups We define a new class of fuzzy groups called fuzzy dot group which is weaker than the standard fuzzy group defined by Rosenfeld and characterize some properties of fuzzy dot groups.

\section{REFERENCES}

[1] 1.J.M.Anthony and .Sherwood, Fuzzy groups redefined J.math.Anal.Appl.69(1979), 124-130.

[2] 2.A.Rosenfeld, Fuzzy groups, J.math.Anal.Appl.35 (1971), 512- 517.

[3] 3.S.Sessa, On fuzzy subgroups and fuzzy ideals under triangular norms, Fuzzy sets and fuzzy systems , 13 , (1984), 95-100.

[4] 4.H.Sherwood, Product of fuzzy subgroups, Fuzzy sets and systems , $11,(1983), 79-89$.
[5] 5.F.I.Sidky and M.Atif Misherf, Fuzzy cossets and cyclic and abelian fuzzy subgroups, Fuzzy sets and systems , 43 , (1991) , 243-250.

[6] 6.A.Solairaju and R.Nagarajan, Characterizations of weaker Fuzzy groups in terms Rosenfeld's Fuzzy groups, Journal of Applied Mathematical Analysis and Applications Vol.4, No. 1-2, 37-43.

[7] 7.R.Nagarajan and A.Solairaju, Some Structure Properties of M- fuzzy groups Applied Mathematical Sciences, Accepted For Publication,2010.

[8] 8.A.Solairaju and R.Nagarajan Some Structure Properties of Q-cyclic Fuzzy group Family, Antarctica Journal of Mathematics, Vol.7.,No.1,2010.

[9] 9..L.A.Zadeh , Fuzzy sets , Inform and control , 8 ,(1965)., 338-353. 